\title{
PREVALENCIA DE BRUCELOSIS CAPRINA EN TRES DISTRITOS DE LA PROVINCIA DE CAÑETE, LIMA
}

\author{
Caprin e Brucellosis in Three Districts of the Cañete Province, Lima \\ Marcel Toledo E. ${ }^{1}$, Alfredo Delgado C. ${ }^{1,3}$, Francisco Suárez A. y Norma Noé M. ${ }^{2}$
}

ReSUMeN

\begin{abstract}
El objetivo del presente estudio epidemiológico fue estimar la prevalencia de brucelosis en cabras criadas bajo un sistema semi-estabulado, en los distritos de San Vicente, Nuevo Imperial y San Luis de la provincia de Cañete, en Lima. Se colectaron muestras de sangre de 385 cabras de tres o más meses de edad, procedentes de 21 hatos y no vacunados contra brucelosis caprina. Hubo cuatro cabras serorreactoras con la prueba Rosa de Bengala, y sólo una fue confirmada mediante la prueba de Fijación de Complemento, dando una prevalencia de brucelosis caprina de $0.26 \pm 0.04 \%$. Esta baja prevalencia podría deberse al efecto positivo del Programa de Control y Erradicación de Brucelosis Caprina conducido por el Ministerio de Salud del Perú (MINSA) y el Servicio Nacional de Sanidad Agraria (SENASA) desde el año 2000 en la provincia de Cañete.
\end{abstract}

Palabras clave: brucelosis, cabras, rosa de bengala, fijación de complemento, SENASA

\section{Abstract}

The objective of the present epidemiological study was to determine the prevalence of brucellosis in raised goats under a barn-feeding system in the districts of San Vicente, Nuevo Imperial and San Luis, Cañete Province, Lima. Blood samples were collected from 385 goats of three or more months of age, not vaccinated against caprine brucellosis and belonging to 21 herds. Results showed four sero-positive goats to the Rose Bengal test and only one to the Complement Fixation test. The prevalence of caprine brucellosis was estimated at $0.26 \pm 0.04 \%$. This low prevalence could be due to the positive effect of the Control and Eradication Program of Caprine Brucellosis conducted by the Peruvian Ministry of Health and the National Service of Agrarian Health (SENASA) since the year 2000 in the province of Cañete.

Key words: brucellosis, goats, Rose Bengal, complement fixation test, SENASA

${ }^{1}$ Clínica de Animales Mayores, ${ }^{2}$ Laboratorio de Medicina Veterinaria Preventiva, Facultad de Medicina Veterinaria, Universidad Nacional Mayor de San Marcos, Lima

${ }^{3}$ E-mail: adelgadoc@unmsm.edu.pe 


\section{INTRODUCCIÓN}

La población caprina en el Perú es de 2’004,374 de cabezas, encontrándose mayor proporción en la Sierra $(68 \%)$ y en la Costa (31\%), que en la selva (1\%). Los departamentos de mayor población caprina son Piura, Ancash, Lima, Ica, Ayacucho y Huancavelica, representando más del $55 \%$ del total nacional. Buena parte de esta población de caprinos son manejados por productores de bajo nivel tecnológico, quienes sin embargo hacen un buen uso de recursos marginales para la alimentación de los animales, entre ellos residuos de cosecha, pastos naturales y especies arbustivas (Arroyo, 1989; INEI, 1994). En algunas zonas del país se cría a estos animales en grandes grupos y con control sanitario limitado, lo que permite una interrelación indiscriminada entre hatos, ocasionando que animales infectados y susceptibles se junten, propiciando la transmisión de enfermedades de diversas etiologías, entre ellas la brucelosis caprina (SENASA, 2004).

La brucelosis es una entidad nosológica infecciosa producida por una bacteria Gram negativa intracelular facultativa del género Brucella, que tiene como principal reservorio a la especie caprina. Es considerada una zoonosis clásica de mecanismo fisiopatológico complejo, donde el hombre es un hospedero accidental (Corbel, 1991; Acosta y Ortiz, 2005).

La brucelosis es una enfermedad que puede transmitirse de los caprinos infectados al hombre. Es conocida también como Fiebre Malta, Fiebre Mediterránea o Fiebre Ondulante. La transmisión puede ser por contacto o por ingestión de productos lácteos contaminados con Brucella, principalmente quesos, representando entonces un riesgo para la Salud Pública. Además, la enfermedad incide directamente en la productividad animal, al ocasionar pérdidas económicas por abortos en las cabras y mermas de hasta $25 \%$ de la producción de leche. No obstante, los esfuerzos por instituir medidas de control y erradicación, la brucelosis es todavía un problema importante para la salud y la economía de muchos países (Cecil, 1996; Acha y Szyfres, 2001; SENASA, 2004).

Por estas razones, el presente estudio epidemiológico buscó estimar la prevalencia de la brucelosis caprina en los distritos de San Vicente, Nuevo Imperial y San Luis de la Provincia de Cañete, Departamento de Lima, con el propósito de conocer el comportamiento de la enfermedad en esta región, y de apoyar la toma decisiones, dentro de lo que pueden ser medidas zoosanitarias pertinentes.

\section{Materiales y Métodos}

\section{Lugar de estudio y animales}

El muestreo del presente estudio fue realizado en hatos caprinos localizados en un área de 4,581 km², en los distritos de sur de Lima, entre los meses de agosto a septiembre del 2004. Se recolectaron muestras de sangre de 385 caprinos hembra de tres o más meses de edad, procedentes de 21 hatos (Cuadro 1), y que no fueron vacunados contra brucelosis caprina. Las muestras se tomaron mediante punción de la vena yugular.

El procesamiento de las muestras de sangre de los caprinos se realizó en el laboratorio del Servicio Nacional de Sanidad (SENASA), Lima, Cañete, y en el Laboratorio Central de SENASA, Lima.

Las cabras eran criadas bajo un sistema semiestabulado y no transhumante, siendo alimentados en su corral por la mañana y llevados a pastoreo por la tarde. Su alimento principal era maíz chala, pastos naturales y rastrojos de cosecha.

\section{Procedimiento metodológico}

Las muestras colectadas, debidamente identificadas, fueron transportadas al laboratorio del SENASA-Cañete para ser procesadas el mismo día de recolección utilizando la prueba tamiz Rosa de Bengala. 
Cuadro 1. Número de hatos y de cabras muestreadas para diagnóstico de brucelosis en distritos de la provincia de Cañete, Lima (agosto a septiembre, 2004)

\begin{tabular}{lcccc}
\hline \multirow{2}{*}{ Distritos } & Hatos & \multicolumn{3}{c}{ Animales muestreados } \\
\cline { 3 - 5 } & Muestreados & Total & Jóvenes & Adultos \\
\hline San Vicente & 9 & 186 & 76 & 110 \\
Nuevo Imperial & 7 & 111 & 26 & 85 \\
San Luis & 5 & 88 & 16 & 72 \\
\hline Total & 21 & 385 & 118 & 267 \\
\hline
\end{tabular}

1Jóvenes: de 3 a 6 meses de edad; Adultos: de 6 ó más meses de edad

Cuadro 2. Muestras positivas a la prueba de aglutinación Rosa de Bengala en animales de la provincia de Cañete, Lima (agosto a septiembre, 2004)

\begin{tabular}{lccc}
\hline Distritos & $\begin{array}{c}\text { Jóvenes } \\
(\mathrm{n}=118)\end{array}$ & $\begin{array}{c}\text { Adultos } \\
(\mathrm{n}=267)\end{array}$ & $\begin{array}{c}\text { Total } \\
(\mathrm{n}=385)\end{array}$ \\
\hline San Vicente & 0 & $3^{1}$ & 3 \\
Nuevo Imperial & 0 & 1 & 1 \\
San Luis & 0 & 0 & 0 \\
\hline
\end{tabular}

${ }^{1}$ Uno de estos animales resultó positivo a la prueba a la prueba de Fijación de Complemento

Las muestras que resultaron positivas, fueron sometidas a la prueba de Fijación de Complemento, que es considerada como prueba confirmatoria por la Organización Mundial de la Salud (OMS/FAO, 1986; Corbel, 1991; Tizard, 2002).

\section{Análisis estadístico}

La prevalencia aparente de la brucelosis caprina fue corregida considerando la sensibilidad (99\%) y especificidad (100\%) de la prueba confirmatoria (Ahlbom y Novell, 1992).

\section{Resultados y Discusión}

Se encontró una prevalencia de $0.26 \pm$ $0.04 \%$ en los tres distritos de la Provincia de Cañete. La muestra positiva fue confirmada como serorreactiva mediante la prueba de Fijación de Complemento, dentro de cuatro muestras halladas como serorreactivas a la prueba Rosa de Bengala (Cuadro 2).

En la provincia de Cañete, el Ministerio de Salud (MINSA) viene llevando a cabo un Programa de Control y Erradicación de 
la Brucelosis Caprina (PCEbc) desde el año 2000 y el SENASA desde el año 2003. En el programa se realizan diversas actividades, entre ellas el registro de hatos, vacunación sistemática de los animales (hembras mayores de tres meses), monitoreo serológico, sacrificio de los animales serorreactores a la prueba confirmatoria y vigilancia epidemiológica; actividades que habrían influenciado el resultado encontrado.

En los años anteriores no se llevaba registro adecuado de los hatos ni de los animales. El PCEbc ha permitido un mejor manejo y control de los animales en las inmunizaciones, lo cual previene la infección en la mayoría de animales susceptibles, y por consiguiente mantiene la infección de campo en niveles bajos. Asimismo, con la discriminación de animales serorreactores y su posterior sacrificio, se está evitando la transmisión de la infección en el hato y su posible persistencia en él.

Adicionalmente, los criadores de Cañete aplican el sistema de crianza semiestabulado y no transhumante, que permite que los animales tengan un lugar fijo, tanto de residencia como de alimentación, con lo cual no hay mayor contacto con criadores de otras regiones. En otros lugares, como en el norte del Perú, hatos de diferentes criadores se juntan durante la comercialización de animales y subproductos lácteos, lo que incrementa el factor de riesgo, y esto podría traducirse en el hallazgo de un mayor número de animales serorreactores. Por otro lado, la zona de Cañete se encuentra en un valle rodeado de cerros y quebradas que actuarían como barrera natural, impidiendo el fácil acceso de criadores de otros distritos y provincias, con hatos posiblemente infectados con Brucella.

No existen estudios epidemiológicos previos en la región, pero los resultados son comparables con trabajos similares realizados en otras zonas. Castagnino et al. (1967) encontraron una prevalencia de $14 \%$ en el depar- tamento de Lima utilizando la prueba de aglutinación en placa, prueba de menor sensibilidad y especificidad que Rosa de Bengala. Escurra (1970) reportó una prevalencia de 43\% en La Libertad y en Ancash, donde la transhumancia es común entre los criadores. En este caso se utilizó la prueba de anillo en leche, la cual se adapta mejor para el diagnóstico de brucelosis en ganado bovino y porque además, es una prueba para la detección de Brucella en muestras colectivas y no individuales (Shimi y Tabatabayi, 1981; Radostits et al.,2002).

El reporte que mejor se aproxima al presente estudio es el realizado por Solé (1999), quien encuentra una prevalencia de $1.7 \%$ en la zona de Chincha, utilizando la prueba de Rosa de Bengala. En esa zona, en forma similar, la crianza de las cabras es semiestabulada y el programa PCEbc se encuentra en marcha. En ese estudio, la prevalencia verdadera podría ser menor si se hubiese empleado la prueba de Fijación de Complemento, toda vez que la primera produce "falsos positivos", especialmente cuando la prevalencia es baja o se practica la vacunación (Acha y Szifres, 2001). Esta misma situación podría ser el caso del estudio de Vargas (1999) en el valle del Chillón, Canta, Lima, quien encontró una prevalencia de $2.8 \%$ en la población caprina. En fechas más recientes, y dentro de las zonas de cobertura del PCEbc, no se encontraron reactores seropositivos en las provincias de Barranca (Garro, 2004) y Huarochirí (Rojas, 204).

El bajo número de animales serorreactores a brucelosis caprina (1/385) encontrado en el presente estudio es un importante hallazgo, al constituirse como herramienta informativa sobre el comportamiento epidemiológico de la brucelosis caprina en los distritos estudiados de la provincia de Cañete; que por otro lado albergan a la mayor población caprina de la provincia. Esto podría contribuir para que las autoridades sanitarias del Estado evalúen el éxito de sus programas establecidos y puedan en su momento declarar el área como libre de la enfermedad. 


\section{Literatura Citada}

1. Acha P, Szyfres B. 2001. Zoonosis y enfermedades transmisibles comunes al hombre y a los animales: bacteriosis y micosis. $3^{\text {a }}$ ed. Washington, EEUU: OPS. p 28-52.

2. Acosta M, Ortiz M. 2005. Brucelosis caprina. MV Rev Cien Vet 21(2): 9-12.

3. Alhbom A, Norell S. 1992. Fundamentos de epidemiología. $3^{a}$ ed. Madrid: Ed. Siglo XXI. p 33-34.

4. Arroyo O. 1989. Producción de caprinos. Lima: Ed. Caligráfica.p 257307.

5. Castagnino D, Huamán M, Matto E. 1967. Epidemiología de la brucelosis. En: Rojas, CM. (ed). IVITA: 30 años de ciencia y tecnología pecuaria. Lima: Universidad Nacional Mayor de San Marcos. p 36.

6. Cecil B. 1996. Tratado de medicina interna. EEUU: McGraw-Hill Interamericana. Vol. II. 20a ed. p 98-120.

7. Corbel MJ. 1991. Brucelosis. En: Laing JA, Brinley Morgan WJ, Wagner WC. (eds). Fertilidad e infertilidad en la práctica veterinaria. $4^{\mathrm{a}}$ ed. España: Ed. Interamericana. p 10-15.

8. Ezcurra E. 1970. Epidemiología de la brucelosis caprina en los departamentos de La Libertad y Ancash. Tesis de Bachiller. Lima: Facultad de Medicina Veterinaria, Univ. Nacional Mayor de San Marcos. 20 p.

9. Garro A. 2004. Prevalencia de la brucelosis caprina de la provincia de Barranca, departamento de Lima. Tesis de Médico Veterinario. Lima: Facultad de Medicina Veterinaria, Univ. Nacional Mayor de San Marcos. 27 p.

10. [INEI] Instituto Nacional de Estadística e Informática. 1994. Censo Na- cional Agropecuario.[Internet] Disponible en: http://www.inei.gob.pe/Web/ resultadocenso.asp. (18/08/2004).

11. OMS/FAO. 1986. Sexto informe del comité mixto de expertos de Brucelosis. Serie de Informes Técnicos 740. Ginebra. $149 \mathrm{p}$.

12. Radostits O, Gay S, Blood D, Hinchacliff $K$. 2002. Medicina veterinaria. $9^{n}$ ed. España: McGraw-Hill. p 1030-1053.

13. Rojas G. 2004. Seroprevalencia de Brucella sp. en caprinos de Huarochirí, Lima. Tesis de Médico Veterinario. Lima: Facultad de Medicina Veterinaria, Univ. Nacional Mayor de San Marcos. 26 p.

14. SENASA. 2004. Situación actual de la brucelosis caprina en el Perú. [Internet] Disponible en: http://www.senasa.gob.pe /sanidadanimal/programazoosanitarios/ brucelosiscaprina.htm (26/09/2004).

15. Shimi A, Tabatabayi AH. 1981. Pathological, bacteriological responses of ewes experimentally infected with Brucella melitensis. Bulletin Office International des Epizooties 93: 14111422.

16. Solé C. 1999. Estudio epidemiológico de la brucelosis caprina en la provincia de Chincha. Tesis de Médico Veterinario. Ica: Facultad de Medicina Veterinaria, Univ. Nacional San Luis Gonzaga de Ica. $26 \mathrm{p}$.

17. Tizard I. 2002. Inmunología veterinaria. 6 ed. México: McGraw-Hill Interamericana. $517 \mathrm{p}$.

18. Vargas M. 1999. Prevalencia de la Brucella melitensis en el valle de Chilón, provincia de Canta, departamento de Lima. Tesis de Médico Veterinario. Lima: Facultad de Medicina Veterinaria, Univ. Nacional Mayor de San Marcos. $28 \mathrm{p}$. 